\title{
Use of a pectus bar for surgical stabilization of cardiopulmonary resuscitation induced flail chest
}

\author{
SungKwang Lee ${ }^{1}$, dohyung kim ${ }^{1}$, and Chee Hoon Lee ${ }^{1}$ \\ ${ }^{1}$ Pusan National University Yangsan Hospital
}

May 6, 2020

\begin{abstract}
A flail chest can occur when cardiopulmonary resuscitation causes extensive rib fractures. Despite successful cardiopulmonary resuscitation, if the flail chest is not treated, the patient may not survive regardless of the correction of the primary condition that caused the cardiac arrest. Therefore, if flail chest persists despite proper conservative management to correct the flail chest, active surgical management is essential. We present a successful surgical treatment with pectus bar for a patient with flail chest, caused by extensive segmental rib fractures sustained during cardiopulmonary resuscitation for a massive pulmonary thromboembolism.
\end{abstract}

Title: Use of a pectus bar for surgical stabilization of cardiopulmonary resuscitation induced flail chest

Running head: Pectus bar insertion for flail chest

Authors: Sung Kwang Lee, M.D., Do Hyung Kim, M.D. Chee Hoon Lee, M.D.

Affiliations: Department of Thoracic and Cardiovascular Surgery, Pusan National University Hospital, South Korea.

Word count: 1343

Corresponding author: Do Hyung Kim, M.D., Department of Thoracic and Cardiovascular Surgery, Pusan National University Yangsan Hospital, Beomeo-ri, Mulgeum-eup, Yangsan, Gyeongsangnam-do, South Korea 626-770, Tel. +82-55-360-2127, Fax. +82-55-360-2127, E-mail. yumccs@nate.com

Abstract

A flail chest can occur when cardiopulmonary resuscitation causes extensive rib fractures. Despite successful cardiopulmonary resuscitation, if the flail chest is not treated, the patient may not survive regardless of the correction of the primary condition that caused the cardiac arrest. Therefore, if flail chest persists despite proper conservative management to correct the flail chest, active surgical management is essential. We present a successful surgical treatment with pectus bar for a patient with flail chest, caused by extensive segmental rib fractures sustained during cardiopulmonary resuscitation for a massive pulmonary thromboembolism.

Key words: cardiopulmonary resuscitation, flail chest, pectus bar insertion

Introduction

A flail chest can be caused during cardiopulmonary resuscitation. In cases where chest wall stability is not achieved with conservative treatment, active surgical correction becomes essential. Conventional wide dissection and multiple rib plating are not recommended for high-risk patients, such as those with pre-existing cardiac conditions. Therefore, a less invasive and surgically safe alternative is needed. Recent reports have 
highlighted the use of a technique using a pectus bar that was originally used in pediatrics, in patients with severe trauma to the anterior chest wall. We found this to be an ideal surgical intervention for such patients.

Case

A 57-year-old woman with Cushing's disease was admitted to the emergency room with seizure and drowsy mentality. A cardiac arrest occurred during the observation period and six cycles of cardiopulmonary resuscitation were performed. The patient was then intubated and underwent mechanical ventilation, and VA-ECMO insertion, following which CT was performed that revealed a massive acute pulmonary thromboembolism and intramural hematoma of aorta (Figure 1). Tissue plasminogen activator infusion was performed immediately after the diagnosis, but the pulmonary embolism did not improve and vital signs were difficult to maintain. On the fourth day of admission, emergency pulmonary thromboembolectomy and hemi-arch replacement of the aorta were performed.

In the post-operative period, spontaneous breathing was induced to wean the patient from the mechanical ventilator. However, this failed due to breathing difficulties accompanied by paradoxical chest movement. Even after 38 days of positive pressure ventilation, the chest wall instability did not improve. Since the conservative treatment failed, a surgical approach was adopted. Chest CT was performed just before the pectus bar insertion, and bilateral segmental fractures were visualized from the 2 nd to 7 th ribs, along with anterior chest wall depression (Figure 2a). Plating of the 12 segmental fractures was considered difficult due to the general condition of the patient, and the pectus bar was surgically inserted under general anesthesia, to maintain anterior chest wall stability. The lower third of the previous mid-sternotomy skin incision was re-incised to relieve the adhesions from the previous surgery, between the anterior chest wall (including the sternum) and the heart (Figure 2b). After the dissection, a pectus bar was inserted through 3-cmlong bilateral skin incisions on the chest (Figure 2c). The surgery was completed after chest stability was confirmed. Chest CT evaluation on the day after the surgery showed chest wall elevation and maintenance of the normal rib cage shape by the pectus bar (Figure 2d). After the surgery, the patient was ventilated using a portable home ventilator, and respiratory rehabilitation was performed. The patient was discharged 4 months post-operatively, after she was able to breathe spontaneously without a respiratory system.

Discussion

Chest compressions performed during cardiopulmonary resuscitation require the application of strong forces on the chest repeatedly. This may lead to a number of secondary injuries. Among these, rib fractures have an incidence of 12.9 to $96.6 \%$ and can result in a flail chest that presents as a paradoxical chest movement. Autopsy results have reported the occurrence of flail chest after cardiopulmonary resuscitation to be common, with an incidence of $14.8 \%(1)$.

When a flail chest occurs, ventilator weaning is often difficult due to the instability of the respiratory work caused by the paradoxical chest movement. If this movement persists before the spontaneous chest wall stabilization, there can be an additional mechanical impact on the contused lung area. Therefore, the treatment of flail chest is important to maintain chest stability. Severe flail chest usually requires mechanical ventilation with PEEP for 7 to 14 days until the paradoxical movement disappears. However, prolonged mechanical ventilation can cause complications such as nosocomial infections and hence, surgical intervention is essential. Although no significant difference was found in the mortality rates between surgical and conservative treatments, the former resulted in reduced pneumonia rate, duration of mechanical ventilation, and ICU stay (2).

The purpose of the surgical treatment of flail chest is to fix the fracture site. However, extensive plate fixation is required with a large incision and wide chest wall dissection. In patients with a history of cardiac arrest, as was true in the present case, increasing the risk of surgery by wide dissection and multiple rib and sternum plating is not desirable. Hence, a less invasive treatment method should be selected to maintain the chest stability.

Surgical treatment with pectus bar insertion is considered to be one of the lesser invasive methods for flail 
chest management and was adopted for our patient. Pacheco et al. (3) reported the stabilization of severe flail chest by using four pectus bars in a patient where segmental fixation was not possible owing to rib fractures. Following this report, chest wall stabilization using pectus bars was employed for cases that were impossible to treat by conventional plating methods (4-6). In the first report, it was used for the purpose of correcting flail chest on one side of the chest wall; however, recently it has been employed for bilateral anterior chest walls. Therefore we considered it to be a suitable treatment for the present case, and it proved to be successful as evidenced by the chest wall stability observed post-operatively along with complete respiratory rehabilitation of the patient.

\section{References}

1. Akkuş M, Utkusavaş A, Hanözü M, Kaya M, Bakir I. Stabilization of Flail Chest and Fractured Sternum by Minimally Invasive Repair of Pectus Excavatum. Thorac Cardiovasc Surg Rep. 2015;4(1):11-3.

2. Schuurmans J, Goslings JC, Schepers T. Operative management versus non-operative management of rib fractures in flail chest injuries: a systematic review. Eur J Trauma Emerg Surg. 2017;43(2):163-8.

3. Pacheco PE, Orem AR, Vegunta RK, Anderson RC, Pearl RH. The novel use of Nuss bars for reconstruction of a massive flail chest. J Thorac Cardiovasc Surg. 2009;138(5):1239-40.

4. Lee SA, Hwang JJ, Chee HK, Kim YH, Lee WS. Flail chest stabilization with Nuss operation in presence of multiple myeloma. J Thorac Dis. 2014;6(5):E43-7.

5. Lee SK, Kang do K. Nuss procedure for surgical stabilization of flail chest with horizontal sternal body fracture and multiple bilateral rib fractures. J Thorac Dis. 2016;8(6):E390-2.

6. Kim JJ, Kim YH, Moon SW, Choi SY, Jeong SC. Nuss procedure for severe flail chest after blunt trauma. Ann Thorac Surg. 2015;99(2):e25-7.

Figure legend Figure 1. Chest CT performed immediately after ECMO insertion showing intramural hematoma of the aorta (yellow arrow) and massive pulmonary thromboembolism (red arrow) Figure 2. Chest CT performed just before the pectus bar insertion with three-dimensional chest wall reconstruction based on the CT data of patients, a) bilateral segmental fractures were seen from the 2nd to 7th ribs with anterior chest wall depression, secondary to flail chest. The segmental resection site is colored blue, b) adhesiolysis (yellow arrow) performed by re-incising the lower third of the previous mid-sternotomy skin incision to relieve the adhesions between the anterior chest wall and the heart, c) pectus bar (green colored bar) was inserted after bilateral 3-cm-long skin incisions on the chest, d) chest wall elevation and normal rib cage shape maintained by the pectus bar 

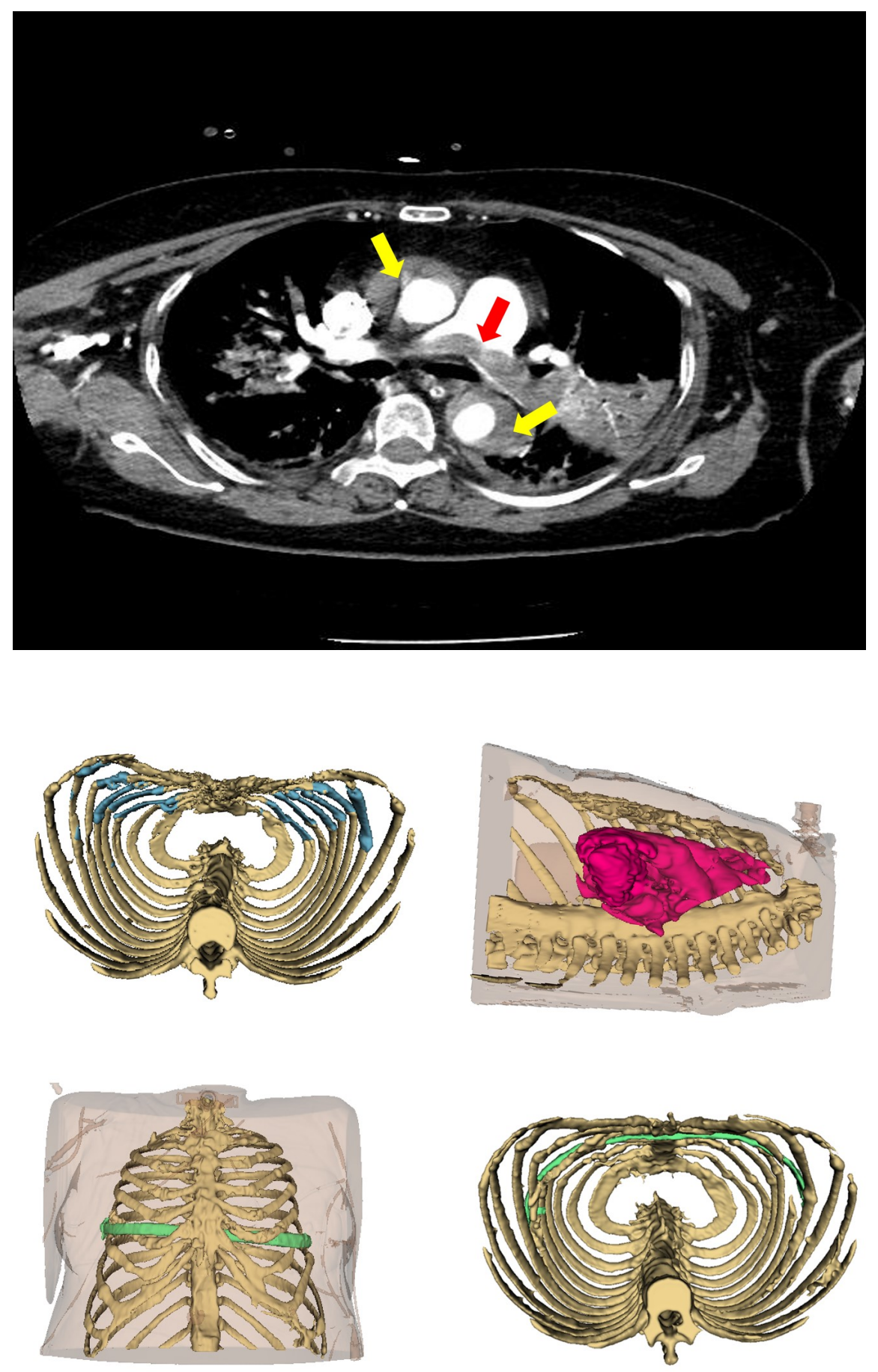